\title{
Una gráfica de control combinada para identificar señales fuera de control en procesos multivariados
}

\author{
A Combined Control Chart for Identifying \\ Out-Of-Control Points in Multivariate Processes
}

\author{
Marroquín-Prado E. \\ División de Estudios de Posgrado e Investigación \\ Instituto Tecnológico de Saltillo \\ E-mail:emarro53@hotmail.com \\ Cantú-Sifuentes M. ${ }^{1}$ \\ División de Posgrado en Ingeniería Industrial \\ Corporación Mexicana de Investigación en Materiales \\ E-mail:mcantu@comimsa.com.mx
}

(Recibido: noviembre de 2006; reevaluado: agosto de 2009; aceptado: julio de 2010)

\section{Resumen}

La gráfica de control $\mathrm{T}^{2}$ de Hotelling se ha empleado ampliamente para identificar señales fuera de control en procesos multivariados. Sin embargo, esta gráfica no es sensible a cambios pequeños en el vector de medias del proceso. En este trabajo se propone una gráfica de control para identificar señales fuera de control. La gráfica propuesta es una combinación de la gráfica $\mathrm{T}^{2}$ de Hotelling, la gráfica $\mathrm{M}$ propuesta por Hayter et al. (1994) y una nueva gráfica basada en Componentes Principales. La combinación de las tres gráficas permite identificar cualquier tipo y magnitud de cambio en el vector de medias del proceso. Por medio de simulación y la Longitud Promedio de Corridas (ARL), se evalúa la eficiencia de la gráfica propuesta. El ARL indica el número promedio de puntos dentro de control antes de encontrar un punto fuera. Los resultados de la simulación muestran que la gráfica propuesta es más sensible que cada una de las tres gráficas en forma individual.

Descriptores: procesos multivariados, $T^{2}$ de hotelling, componentes principales, ARL, gráfica de control M.

\begin{abstract}
The Hotelling's $T^{2}$ control chart is widely used to identify out-of-control signals in multivariate processes. However, this chart is not sensitive to small shifts in the process mean vector. In this work we propose a control chart to identify out-of-control signals. The proposed chart is a combination of Hotelling's $T^{2}$ chart, $M$ chart proposed by Hayter et al. (1994) and a new chart based on Principal Components. The combination of these charts identifies any type and size of change in the process mean vector. Using simulation and the Average Run Length $(A R L)$, the performance of the proposed control chart is evaluated. The ARL means the
\end{abstract}

\footnotetext{
${ }^{1}$ Mediante proyecto especial con la Universidad Autónoma Agraria Antonio Narro.
} 
DOI: http://dx.doi.org/10.22201/fi.25940732e.2010.11n4.039

Una gráfica de control combinada para identificar señales fuera de control en procesos multivariados

average points within control before an out-of-control point is detected, The results of the simulation show that the proposed chart is more sensitive that each one of the three charts individually.

Keywords: Multivariate processes, hotelling's $T^{2}$, principal components analysis, $A R L, M$ control chart.

\section{Introducción}

Cuando la calidad de un producto manufacturado se controla mediante una sola característica, la gráfica de control de Shewhart es ampliamente utilizada (Lowry et al., 1995). Sin embargo, cuando es necesario controlar la calidad del producto mediante $p>1$ características de calidad, es conveniente considerarlas simultáneamente; ya que el uso de $p$ gráficas de control univariadas (una por variable) de Shewhart es un enfoque no razonable, siendo que no considera la correlación que usualmente existe entre las variables de este tipo (Nedumaran et al., 1998). Además, sería complicado controlar el error tipo I global (Nedumaran et al., 1998; Hayter et al., 1994).

Harold Hotelling inicia en 1947 el control estadístico de procesos multivariados, proponiendo una gráfica de control que actualmente lleva su nombre. La construcción de ésta se basa en la cantidad $T^{2}$, que en su versión poblacional se puede definir como sigue; siendo una población normal $p$-variada con vector de medias $\mu$ y matriz de covarianzas $\Sigma>0$, la variable aleatoria $T^{2}$ de Hotelling queda definida mediante la expresión:

$$
T^{2}=(X-\mu)^{\prime} \Sigma^{-1}(X-\mu)
$$

Esta forma cuadrática en $\Sigma^{-1}$ mide la distancia estadística entre cualquier vector aleatorio $X_{i}$ de la población y su vector de medias. Cuando $\mu$ y $\Sigma$ son conocidos, se puede demostrar (Johnson et al., 1998) que $T^{2}$ se distribuye como una $\chi^{2}$ cuadrada con $p$ grados de libertad. Cuando, como generalmente ocurre, tanto $\mu$ como $\Sigma$ son desconocidas es deseable contar con una versión muestral de $T^{2}$. Siendo $X_{1}, \ldots X_{n}$ una muestra de $n$ vectores aleatorios que provienen de una población normal $p$-variada con media $\mu$ y matriz de covarianzas $\Sigma$, éstos son estimados por $\bar{X}$ y $S$, respectivamente, $y$

$$
T^{2}=(X-\bar{X})^{\prime} S^{-1}(X-\bar{X})
$$

define la versión muestral de $T^{2}$ en (1). En este caso se tiene que

$$
T^{2} \sim\left[\frac{p(n+1)(n-1)}{n(n-p)}\right] F_{(p, n-p)}
$$

De aquí en adelante, cuando se mencione a la $T^{2}$ nos referiremos al estadístico en (2).

La implementación de una gráfica de control se realiza en dos fases. En la fase I se define la base histórica de datos o distribución de referencia. A partir de ésta se estiman los parámetros pertinentes. En la fase II se evalúa el comportamiento actual del proceso con respecto a la distribución de referencia. Para construir la gráfica de control $T^{2}$ de Hotelling, de la distribución de referencia se calculan $\bar{X}$ y $S$. El límite de control, $L C$, se define para un nivel de significancia dado $\alpha$ como el cuantil $1-\alpha$, de la distribución en (3). Para cada producto seleccionado del proceso se miden las $p$ características de calidad y se obtiene su $T^{2}$, si este valor es menor que el $L C$ el proceso se declara dentro de control, caso contrario se declara fuera de control indicando que el proceso se aleja de la distribución de referencia y es deseable identificar las causas de la señal de fuera de control. La gráfica $T^{2}$ controla cambios en el vector de medias suponiendo que la matriz de covarianzas del proceso es igual a la matriz de covarianzas de la distribución de referencia. Para consultar los detalles técnicos referimos al lector a Mason et al. (2001).

Aunque actualmente la gráfica de control $T^{2}$ es una de las más usadas en el CEP multivariado (Umit et al., 2001), (Prins et al., 1997), ésta tiene las siguientes desventajas:

1) La gráfica identifica señales fuera de control, pero no identifica las variables causantes de la señal,

2) Es insensible a cambios pequeños en el vector de medias del proceso (Lowry et al., 1992, 1995),

3) Es insensible a cambios individuales en una componente o un subconjunto de ellas (Hawkins, 1991) y

4) Pierde sensibilidad a medida que el número de variables, $p$, se incrementa (Ye et al., 2006).

Un enfoque utilizado en el CEP multivariado para detectar señales fuera de control e identificar las variables causantes de éstas, es usar Componentes Principales $(C P)$ y el estadístico $T^{2}$ (Jackson, 1991). Asumiendo que se tienen $p$ variables $x_{1}, x_{2}, \ldots x_{p}$, para cada uno de $n$ vectores aleatorios, el i-ésimo componente principal 
DOI: http://dx.doi.org/10.22201/fi.25940732e.2010.11n4.039

Marroquín-Prado E. y Cantú-Sifuentes M.

$Z_{i}=a_{i 1} x_{1}+a_{i 2} x_{2}+\ldots+a_{i p} x_{p}$ es una combinación lineal de las $p$ variables $x_{1}, x_{2}, \ldots x_{p}$ y tiene varianza $\lambda_{i}$. Los coeficientes $a_{i 1}, a_{i 2}, \ldots, a_{i p}$ son dados por el i-ésimo vector propio y $\lambda_{i}$ es el i-ésimo valor propio de la matriz de covarianzas 0 de correlaciones de modo que $\lambda_{1} \geq \lambda_{2} \geq \ldots \geq \lambda_{p}$ (Johnson et al., 1998).

El estadístico $T^{2}$ puede representarse en forma equivalente en función de los componentes principales como:

$$
T^{2}=\sum_{i=1}^{p} \frac{Z_{i}^{2}}{\lambda_{i}}
$$

donde $Z_{i}=a_{i}\left(X_{i}-\bar{X}\right)$ es el i-ésimo componente principal calculado sobre la base de los datos centralizados. Cuando la gráfica de control $T^{2}$ en (4), identifica una señal fuera de control, al menos un $Z_{1}$ está fuera de control, por lo tanto, el componente principal $\left|Z_{i}\right|$ máximo es usado para identificar las variables fuera de control. Al respecto, existen al menos dos posturas diferentes; por una parte, Hawkins (1991) menciona que los componentes principales no tienen una interpretación práctica en función de las variables originales; por la otra, Chen et al., (2001), afirman que en algunos casos la combinación de variables de un componente principal proporciona una descripción más clara del proceso que las variables individuales.

Para detectar cambios pequeños en el vector de medias y así obtener una sensibilidad mayor que la gráfica $T^{2}$ se han propuesto las gráficas de control multivariadas de: Promedios Móviles Ponderados Exponencialmente, MEWMA y Sumas Acumuladas, MCUSUM. Sin embargo, estas gráficas no son sensibles a cambios grandes en el vector de medias (Montgomery, 2001). Por lo anterior, algunos expertos recomiendan utilizar en conjunto una gráfica $T^{2}$ de Hotelling con una gráfica MEWMA o MCUSUM para detectar cualquier magnitud de cambio en el vector de medias del proceso.

Hayter et al. (1994) desarrollaron una gráfica de control para identificar señales fuera de control y las variables causantes de éstas. La gráfica está basada en el estadístico $M$ definido como:

$$
M=\max _{j} \frac{\left|x_{i j}-\bar{x}_{j}\right|}{s_{i j}}
$$

donde $x_{i j}$ es la $j$-ésima componente del $i$-ésimo vector observado, $\bar{x}_{J}$ y $s_{i j}$ son la j-ésima componente del vector de medias estimado y el elemento $j j$ de la matriz de covarianzas estimada, respectivamente. Si el estadístico
$M$ es mayor que un valor crítico $\mathrm{Cr}$, el proceso se declara fuera de control y se recomienda examinar $p$ gráficas de control univariadas para identificar las variables causantes de la irregularidad. Cr es una función de la matriz de covarianzas de la distribución de referencia y su valor se puede obtener de tablas disponibles en Odeh et al. (1982) o estimarlo mediante simulación.

La desventaja de este enfoque es que si en el vector observado del proceso se genera un cambio en las variables en sentido opuesto al identificado en la distribución de referencia, el estadístico $M$ no detectará la señal fuera de control (Chen et al., 2001).

Lo anterior, se puede observar en la figura 1 que muestra una correlación positiva entre las variables. Si un vector se mueve en sentido opuesto al indicado en la distribución de referencia (correlación positiva), área B, el punto no es detectado por los límites de control de la gráfica $M$ que son las líneas en puntos gruesos de la figura 1.

Considerando las desventajas que la gráfica $T^{2}$ tiene para identificar pequeños cambios en el vector de medias (Lowry et al., 1992) e identificar cambios individuales en una componente o un subconjunto de componentes del vector de medias (Hawkins, 1991) y la desventaja que la gráfica $M$ tiene para identificar cambios en las variables en sentido opuesto al identificado en la distribución de referencia, Chen et al. (2001), en este trabajo se propone una gráfica de control para identificar cualquier tipo y magnitud de cambio en el vector de medias.

La gráfica de control propuesta está basada en un nuevo estadístico llamado estadístico $Y$, definido como el máximo de los valores estándar absolutos de los componentes principales del vector aleatorio $X_{i}$.

Al combinar esta gráfica con las gráficas $T^{2}$ y $M$, se mejora el desempeño en la detección de señales fuera de control. Una gráfica de control basada únicamente en el estadístico $Y$, es más sensible que las gráficas $T^{2}$ y $M$, para detectar pequeños cambios en el vector de medias del proceso. Por ejemplo, como se comentará posteriormente, el área A en la figura 1 es una zona para la cual las gráficas $T^{2}$ y $M$ no detectarán puntos fuera de control, pero la gráfica $Y$ si lo hará.

Al considerar en forma simultanea los estadísticos $T^{2}, M y Y$, se obtiene una nueva gráfica de control combinada que llamaremos gráfica $T M Y$, la cual tiene el propósito de incrementar la sensibilidad para detectar cualquier magnitud de cambio en el vector de medias del proceso. Se simula un proceso bajo diferentes escenarios de operación y se utiliza $A R L$ como criterio de desempeño de la gráfica propuesta. Los resultados 
muestran que la gráfica TMY responde más rápidamente que cada una de las tres gráficas en forma individual.

\section{Gráfica de control $Y$}

De acuerdo con Jackson (1991), cuando la gráfica de control $T^{2}$ identifica una señal fuera de control, el componente principal en (4) es usado para identificar las variables fuera de control.

A diferencia de este enfoque, en esta sección se propone una nueva gráfica para identificar señales fuera de control, basada en el estadístico $Y$ definido como el máximo del valor absoluto de los $p$ componentes principales estandarizados, esto es:

$$
Y=\operatorname{Max}_{i}\left|Z_{i}\right|
$$

Para un proceso normal multivariado, los componentes principales $Z_{i}$ en (4), se distribuyen como una normal estándar (Johnson et al., 1998). Utilizando teoría elemental de transformaciones, la función de densidad de probabilidad del estadístico $Y$ se expresa en forma general como:

$$
f_{Y}(y)=p\left[2 F_{Z}(y)-1\right]^{p-1} 2 f_{z}(y),
$$

donde $F_{z}(y)$ y $f_{z}(y)$ son la función de distribución acumulada y la función de densidad de probabilidad de $Z$, respectivamente, por lo que la ecuación 6 para el caso normal se reduce a:

$$
f_{Y}(y)=p[2 \Phi(z)-1]^{p-1} 2 \phi(z)
$$

Para calcular el límite de control, $L C$, del estadístico $Y$ para un error tipo I de tamaño $\alpha$, hay que encontrar el valor de $L C$ tal que la integral de la función $f_{Y}(y)$ en (7) sea 1- $\alpha$, esto es, $P(Y \leq L C)=1-\alpha$. En el caso de que los datos no se distribuyan en forma normal multivariada, se pueden utilizar técnicas de remuestreo para estimar el $L C$ de $Y$. El procedimiento para construir la gráfica de control $Y$ es el siguiente:

1. Estimar los parámetros del proceso, $\mu$ y $\Sigma$ a partir de la distribución de referencia.

2. Calcular el límite de control, $L C$, de $Y$.

3. Para cada nueva observación calcular el estadístico $Y$.

4. Si el estadístico $Y$ es menor que el $L C$, el proceso está dentro de control.

5. Si el estadístico $Y$ es mayor o igual al $L C$, el proceso está fuera de control.
En la siguiente sección se presenta un ejemplo para mostrar, mediante simulación, cómo se desempeña una gráfica de control $Y$. A la vez se compara su sensibilidad con las gráficas $T^{2}$ y $M$.

\section{Desempeño de la gráfica $Y$, y comparación de la sensibilidad de las gráficas $T^{2}, M$ y}

Para comparar el desempeño de la gráfica $Y$ con las gráficas $T^{2}$ y $M$, se utiliza el ejemplo propuesto por Hayter et al. (1994), en el cual se supone tener un proceso normal bivariado con $\mu$ y $\Sigma$ conocidos. Dada la matriz $\Sigma$, se calculan los vectores y valores propios. Con los primeros se pueden construir los componentes principales mientras que los segundos proporcionan las varianzas respectivas de los mismos. En un ambiente de simulación se generan $m$ vectores aleatorios, $\mathrm{m}$ grande, de donde se pueden estimar los valores medios de los p componentes principales. Se calculan los límites de control para las tres gráficas considerando un error tipo I de 0.0027. El límite de control para la gráfica $T^{2}$ es el valor de $\chi^{2}$ con dos grados de libertad y es igual a $L C=11.8290$. El límite de control para la gráfica $M$ se obtiene de la forma que lo propone Hayter et al. (1994). De la matriz de correlaciones obtenida de $\Sigma$, se simula el proceso con $m=100,000$ réplicas, proporcionando un $C r=L C=3.1729$. Para calcular el límite de control para la gráfica $Y$, basta integrar la función en (7) tal que $P(Y \leq L C)=1$ - $\alpha$. Realizando este análisis y considerando $p=2$ variables, el límite de control para el estadístico $Y$ es 3.2049.

En la figura 1, la elipse representa el límite de control para el estadístico $T^{2}$. Reescalando a las variables originales, el rectángulo delimitado por líneas con puntos grandes representa los límites de control para el estadístico $M$ y el rectángulo inclinado delimitado por líneas con puntos pequeños, representa los límites de control para el estadístico $Y$.

Se generan 1000 vectores aleatorios, los cuales se representan en la figura 1 en conjunto con las áreas críticas bajo las cuales las tres gráficas, $T^{2}, M y Y$, indican señales fuera de control. Para cualquier vector que se encuentre en el área $B$, la gráfica $T^{2}$ indicará una señal fuera de control, pero no lo hará la gráfica $M$. Por el contrario, todo aquel vector que se encuentre en las áreas $\mathrm{C}$ y $\mathrm{F}$, la gráfica $M$ indicará una señal fuera de control, pero la gráfica $T^{2}$ no lo hará. No obstante, estas diferencias, Hayter et al., (1994) afirman que la eficiencia de ambas gráficas para detectar señales fuera de control, cuando el proceso se encuentra dentro de control, es muy similar en términos de ARL. De la misma figura, se observa que las gráficas $T^{2}$ y $Y$ son más sensibles que 


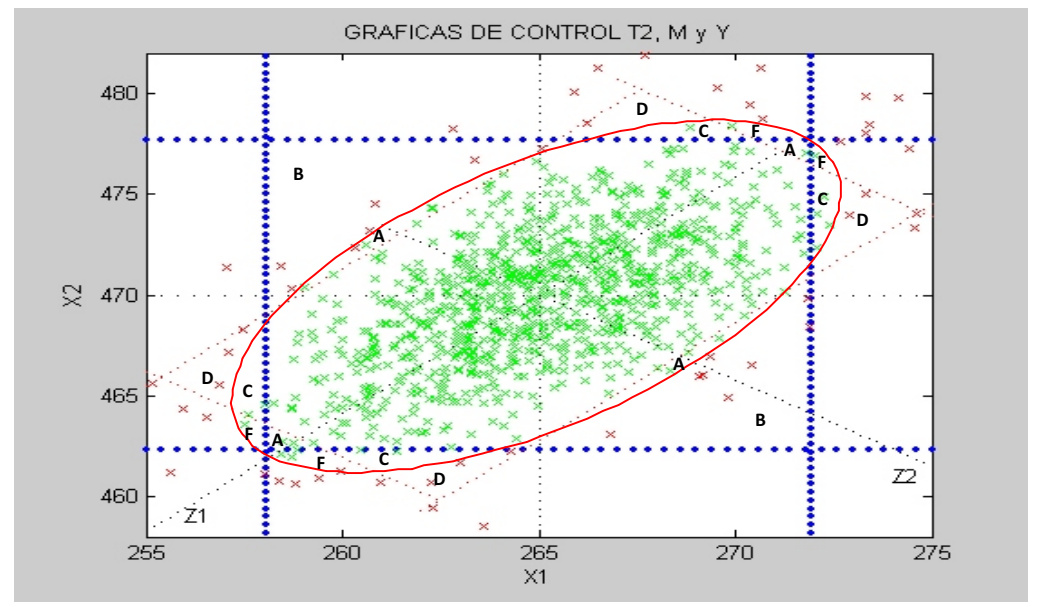

Figura 1. Regiones críticas para los estadísticos $T^{2}, M y Y$

la gráfica $M$ para detectar cambios en las variables en dirección opuesta al identificado en la distribución de referencia (área B). En este sentido, se postula que $Y$ es más eficiente que $T^{2}$ para detectar pequeños cambios en el vector de medias (área A). Por otro lado, se postula que la gráfica $M$ es más eficiente que las gráficas $T^{2}$ y $Y$ para detectar cambios en las variables cuando éstas se incrementan o disminuyen en una misma dirección, áreas C y D.

Para evaluar la sensibilidad de cada gráfica de control y verificar los postulados arriba enunciados, se usa el ejemplo mencionado anteriormente y se realiza una simulación con el proceso bajo control y fuera de control.

Para simular el proceso fuera de control se utilizan ocho diferentes escenarios de cambio en el vector de medias. La magnitud del cambio en el proceso, se mide por medio del parámetro de no centralidad $\lambda$ (Lowry et al., 1992), el cual se expresa como:

$$
\lambda=\left[\left(X_{i}-\mu\right)^{\prime} \Sigma^{-1}\left(X_{i}-\mu\right)\right]^{1 / 2}
$$

Para cada uno de los escenarios, la simulación se realiza con 50,000 vectores individuales. Las tablas 1 y 2 muestran los ARL cuando las medias de las dos variables se incrementan en la misma dirección y cuando las medias de las dos variables se mueven en dirección opuesta (la correlación positiva esperada entre las variables cambia), respectivamente. Cuando un proceso se encuentra bajo control, es deseable que su ARL sea máximo y cuando el proceso está fuera de control, es deseable que su ARL sea mínimo para que identifique más rápidamente las condiciones fuera de control (Sheu et al., 2003-2004).

Tabla 1. Comportamiento de ARL para los estadísticos $T^{2}$, M y (Medias se incrementan en una misma dirección)

\begin{tabular}{ccccccccccc}
\hline Estadístico & \multicolumn{10}{c}{$\boldsymbol{9}$} \\
\cline { 2 - 10 } & 0.00 & 0.25 & 0.50 & 1.00 & 1.50 & 2.00 & 3.00 & 4.00 & 5.00 \\
\hline$T^{2}$ & $\mathbf{3 7 1 . 0 7}$ & 249.90 & 228.31 & 65.78 & 22.52 & 9.07 & 2.54 & 1.29 & 1.04 \\
$M$ & 369.04 & $\mathbf{2 2 7 . 2 7}$ & $\mathbf{1 6 9 . 4 9}$ & $\mathbf{5 2 . 9 1}$ & $\mathbf{1 6 . 8 3}$ & $\mathbf{7 . 1 4}$ & $\mathbf{2 . 2 5}$ & $\mathbf{1 . 2 3}$ & $\mathbf{1 . 0 3}$ \\
$Y$ & 371.74 & 277.77 & 232.55 & 66.66 & 22.47 & 8.83 & 2.37 & 1.24 & 1.03 \\
\hline
\end{tabular}

Tabla 2. Comportamiento de ARL para los estadísticos $T^{2}, M$ y (Medias se incrementan en dirección opuesta)

\begin{tabular}{cccccccccc}
\hline Estadístico & \multicolumn{10}{c}{$\boldsymbol{\lambda}$} \\
\cline { 2 - 10 } & 0.00 & 0.25 & 0.50 & 1.00 & 1.50 & 2.00 & 3.00 & 4.00 & 5.00 \\
\hline$T^{2}$ & $\mathbf{3 7 1 . 0 7}$ & $\mathbf{2 9 0 . 6 8}$ & 185.87 & 57.67 & 23.26 & 8.89 & 2.47 & 1.30 & 1.04 \\
$M$ & 369.04 & 304.87 & 259.06 & 129.20 & 77.88 & 42.62 & 14.28 & 5.69 & 2.65 \\
$Y$ & 371.74 & 308.64 & $\mathbf{1 8 0 . 5 0}$ & $\mathbf{5 5 . 4 9}$ & $\mathbf{2 2 . 0 4}$ & $\mathbf{8 . 2 9}$ & $\mathbf{2 . 2 8}$ & $\mathbf{1 . 2 6}$ & $\mathbf{1 . 0 3}$ \\
\hline
\end{tabular}


De los resultados de la simulación (tablas 1 y 2), cuando el proceso está bajo control, no existe una gráfi$\mathrm{ca}$, de las tres analizadas, que sea más sensible que las otras dos. Este resultado es congruente con el encontrado por Hayter et al. (1994) quienes reportan que cuando el proceso está bajo control, no hay una diferencia clara entre la sensibilidad de las gráficas $T^{2}$ y $M$. Cuando el proceso está fuera de control con las medias de las variables incrementándose en una misma dirección (tabla 1), la gráfica $M$ responde más rápidamente que las otras dos. Cuando el proceso está fuera de control con las medias de las variables cambiando en sentido opuesto (tabla 2), las gráficas $T^{2}$ y $Y$ responden más rápidamente que la gráfica $M$, siendo mejor la respuesta de $Y$.

Considerando los resultados presentados en el análisis anterior y tomando como base al ARL, se concluye que ninguna gráfica de control es más eficiente que las otras dos bajo todas las condiciones de operación simuladas. Se observa que la gráfica $Y$ resulta ser más sensible para detectar cambios en las medias en sentido opuesto al observado en la distribución de referencia.

\section{Gráfica de control combinada}

Con el propósito de disponer de un método de control que sea más sensible a cualquier condición de operación de un proceso, se propone una gráfica de control basada en la combinación de los tres estadísticos; $T^{2}, M$ y $Y$. De acuerdo a los estadísticos utilizados, la gráfica propuesta se denomina gráfica de control combinada TMY. El objetivo de la gráfica de control TMY es incrementar la sensibilidad para identificar cualquier tipo y magnitud de cambio en el vector de medias. El procedimiento para desarrollar la gráfica de control TMY es el siguiente:
1. Estimar los parámetros del proceso $\mu$ y $\Sigma$ a partir de la distribución de referencia.

2. Calcular los $L C$ para cada una de las tres gráficas de control.

3. Para cada nueva observación calcular los tres estadísticos.

4. Si cada estadístico es menor a su $L C$ correspondiente, el proceso está dentro de control.

5. Si al menos un estadístico es mayor o igual a su $L C$ correspondiente, el proceso está fuera de control.

Para mostrar cómo se construye la gráfica de control propuesta, se considera el caso de monitorear y controlar la calidad dimensional de un subensamble en un proceso automotriz para el cual se controlan $p=8$ variables críticas que se correlacionan entre sí. Del proceso de manufactura se identifica la distribución de referencia y se comprueba que los datos siguen una distribución normal multivariada con $\mu$ y $\Sigma$ estimados por $\bar{X}$ y $S$, respectivamente. Dada la matriz de varianzas se calculan los componentes principales. Simulando el proceso se estiman las medias de los componentes principales. Los límites de control para cada estadístico están basados en un error tipo I de tamaño 0.0027. El límite de control para el estadístico $T^{2}$ es el valor de $\chi^{2}$ con ocho grados de libertad y es igual a 23.5744. De una simulación con $n=100,000$ réplicas, el límite de control para el estadístico $M$ es 3.4943. De la función (7) para $p=8$ variables, el límite de control para el estadístico $Y$ es 3.5843. La figura 2 muestra una gráfica de control combinada TMY, donde se observan siete señales fuera de control. Las observaciones 11,14, 28 y 30 son detectadas sólo por la gráfica de control $T^{2}$, la observación 2 es detectada por las gráficas $T^{2}$ y $M$, la observación 4 es detectada por las gráficas $T^{2}$ y $Y$, y la observación 26 sólo es detectada por la gráfica $Y$.

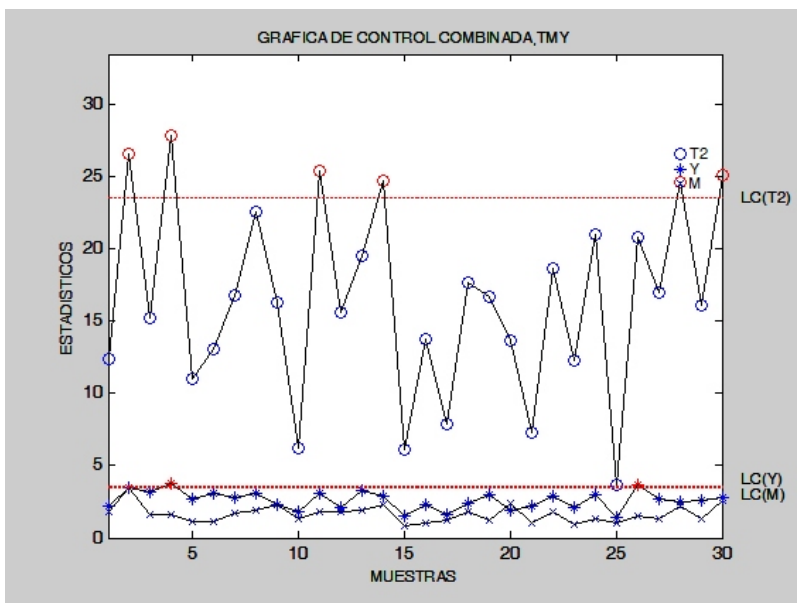

Figura 2. Gráfica de control combinada, TMY 
DOI: http://dx.doi.org/10.22201/fi.25940732e.2010.11n4.039

Marroquín-Prado E. y Cantú-Sifuentes M.

Tabla 3. Comportamiento de $A R L$ para las gráficas $T^{2}, M$ y $Y$ y la gráfica combinada $T M Y$

\begin{tabular}{cccccccccc}
\hline Estadístico & \multicolumn{10}{c}{$\lambda$} & \multicolumn{1}{c}{$\boldsymbol{\lambda}$} \\
\cline { 2 - 9 } & 0.00 & 0.25 & 0.50 & 1.00 & 1.50 & 2.00 & 3.00 & 4.00 \\
\hline$T^{2}$ & 386.19 & 366.39 & 306.28 & 138.40 & 63.23 & $\mathbf{2 8 . 2 1}$ & $\mathbf{6 . 1 0}$ & $\mathbf{2 . 1 2}$ & $\mathbf{1 . 2 4}$ \\
$M$ & 371.45 & 377.98 & 370.96 & 326.64 & 277.32 & 246.54 & 170.00 & 117.05 & 74.91 \\
$Y$ & $\mathbf{3 6 9 . 6 1}$ & $\mathbf{2 8 0 . 3 2}$ & $\mathbf{2 1 9 . 9 5}$ & $\mathbf{1 0 5 . 9 5}$ & $\mathbf{5 6 . 4 2}$ & 29.26 & 8.29 & 3.09 & 1.62 \\
$T M Y$ & $\mathbf{1 5 5 . 4 6}$ & $\mathbf{1 3 7 . 3 2}$ & $\mathbf{1 1 6 . 4 9}$ & $\mathbf{6 2 . 8 9}$ & $\mathbf{3 4 . 2 4}$ & $\mathbf{1 7 . 7 2}$ & $\mathbf{4 . 8 8}$ & $\mathbf{1 . 9 4}$ & $\mathbf{1 . 2 0}$ \\
\% de desempeño & 57.94 & 51.01 & 47.19 & 40.64 & 39.31 & 37.19 & 20 & 8.49 & 3.23 \\
\hline
\end{tabular}

\section{Evaluación de la sensibilidad de la gráfica de control combinada $T M Y$}

Para evaluar la sensibilidad de la gráfica de control $T M Y$, se corre una simulación para el ejemplo del subensamble automotriz, considerando un cambio en dirección opuesta en las medias de dos variables, cuya correlación bajo condiciones normales de operación del proceso es positiva. Como antes, el cambio se mide en función de $\lambda$. Los resultados se muestran en la tabla 3.

Los resultados de la tabla 3 muestran que para pequeños valores de $\lambda, \lambda<2$, la gráfica $Y$ responde más rápidamente que las otras dos, y para valores grandes de $\lambda$, la gráfica $M$ responde mejor que las otras. Si se observa el ARL de la gráfica de control combinada TMY, ésta responde más rápidamente que cada una de las otras tres en forma individual. La última fila de la tabla 3 muestra el porcentaje de desempeño de la gráfica TMY, con respecto a la mejor de las tres gráficas. Los porcentajes muestran que para pequeños y moderados valores de $\lambda$, la gráfica $T M Y$ es más sensible que las otras tres. Para cambios grandes, $\lambda>3$, el desempeño de TMY no es muy significativo.

La gráfica de control combinada incrementa la sensibilidad, pero desafortunadamente incrementa la tasa de falsas alarmas, sobre todo, cuando el proceso está bajo control. Sin embargo, como algunos autores lo plantean, la eficiencia de una gráfica de control combinada debe sobrepasar las pérdidas, debido al incremento en las falsas señales fuera de control (Wu, 1994-1995). Cuando los costos de la mala calidad en ambientes de manufactura exigentes como la industria automotriz, sobrepasan los costos de detener el proceso e investigar las causas de las señales fuera de control, la gráfica de control combinada TMY es una buena alternativa para mantener la competitividad.

\section{Conclusiones}

En este trabajo se presenta una nueva gráfica de control, la cual consiste en una combinación de las gráficas $T^{2}$,
$M y Y$. La gráfica propuesta recibe el nombre de gráfica de control combinada TMY. El objetivo de ésta es incrementar la sensibilidad para detectar cualquier tipo y magnitud de cambio en el vector de medias del proceso. El ARL de la gráfica de control TMY indica que ésta responde más rápidamente que cada una de las tres gráficas en forma individual. El uso de gráficas de control combinadas como es el caso de la gráfica TMY, incrementa la sensibilidad de las gráficas, pero desafortunadamente incrementa la tasa de señales fuera de control.

En la introducción se menciona que algunos expertos proponen el uso combinado de una gráfica $T^{2}$ Hotelling con una gráfica MEWMA o MCUSUM para detectar cualquier magnitud de cambio en el vector de medias del proceso, lo anterior también incrementa la tasa de señales fuera de control. Sin embargo, cuando los costos por paros de líneas de producción, defectos, retrabajos y garantías son prohibitivos, como en los ambientes de manufactura del sector automotriz, la gráfica de control combinada TMY es una buena estrategia de monitoreo y control dimensional de los productos.

\section{Referencias}

Chen K.H, Boning D.S., Welsch R.E. Multivariate Statistical Process Control and Signature Analysis Using Eigenfactor Detection Methods. On: The $33^{\text {rd }}$ Symposium on the Interface of Computer Science and Statistics, Costa Mesa Ca., 2001.

Hawkins D.M. Multivariate Quality Control Based on Regression-Adjusted Variables. Technometrics, 33(1):61-75, 1991.

Hayter A.J., Tsui K.L. Identification and Quantification in Multivariate Quality Control Problems. Journal of Quality Technology, 26(3):197-208, 1994.

Jackson J.E. A User's Guide to Principal Components. John Wiley and Sons, Inc. 1991.

Johnson R.A., Wichern D.W. Applied Multivariate Statistical Analysis. Prentice Hall. 1998.

Lowry A.C., Montgomery D.C. A Review of Multivariate Control Charts. IIE Transactions, 27(6):800-810, 1995. 
Lowry A.C., Woodall H.W. A Multivariate Exponentially Weighted Moving Average Control Chart. Technometrics, 34(1):46-53, 1992.

Mason L.R., Young C.J. Multivariate Statistical Process Control with Industrial Applications. ASA. SIAM. 2001.

Mason L.R., Tracy D.N., Young C.J. A Practical Approach for Interpreting Multivariate $\mathrm{T}^{2}$ Control Chart Signals. Journal of Quality Technology, 29(4):396-406, 1997.

Montgomery D.C. Introduction to Statistical Quality Control. New York. John Wiley and Sons. 2001.

Nedumaran G., Pignatiello J.J. Jr. Diagnosing Signals from $\mathrm{T}^{2}$ and $X^{2}$ Multivariate Control Charts. Quality Engineering, 10(4):657-667, 1998.

Odeh R.E. Tables of Percentage Points of the Distribution of the Maximum Absolute Value of Equally Correlated
Normal Random Variables. Communications in Statistics-Simulation and Computation, (11):65-87, 1982.

Prins J., Mader D. Multivariate Control Charts for Grouped and Individual Observations. Quality Engineering, 10(1): 49-57, 1997.

Sheu S.H., Lin T.Ch. The Generally Weigthed Moving Average Control Chart for Detecting Small Shifts in the Process Mean. Quality Engineering, 16(2):209-231, 2003-2004.

Umit F., Cigdem A. Multivariate Quality Control: A Historical Perspective. Yilditz Technical University. 2001. Pp. 54-65.

$\mathrm{Wu}$ Z. An Enhanced X Chart for Detecting Mean Shift. Quality Engineering, 7(2):345-356, 1994-1995.

Ye N., Parmar D., Borror M.C. A Hybrid SPC Method with the Chi-Square Distance Monitoring Procedure for Large-scale, Complex Process Data. Quality and Reliability Engineering International, (22):393-402, 2006.

\section{Semblanza de los autores}

Eduardo Marroquin-Prado. Se graduó en el Instituto Tecnológico de Saltillo como ingeniero industrial. En 1981, obtuvo su maestría en ingeniería industrial por Oregon State University. En 2008, el grado de doctor en ingeniería industrial y de manufactura en la Corporación Mexicana de Investigación en Materiales (COMIMSA) en Saltillo, Coahuila. México. Ha realizado proyectos en diversas áreas de la ingeniería industrial en empresas manufactureras de la región. Actualmente trabaja como profesor investigador en el Instituto Tecnológico de Saltillo.

Mario Cantú-Sifuentes. Obtuvo su licenciatura en ingeniería industrial en el Instituto Tecnológico de Saltillo en 1976. Obtuvo el grado de maestría en ciencias en estadística por la Universidad Autónoma Agraria Antonio Narro y el grado de doctor en ciencias en estadística por el Colegio de Posgraduados. De 1977 a 1983, realizó diversos trabajos en la iniciativa privada y dependencias gubernamentales. Desde 1985, es profesor-investigador en el Departamento de Estadística de la Universidad Autónoma Agraria Antonio Narro. 